\title{
PARACOCCIDIOMICOSIS EN EL SISTEMA NERVIOSO CENTRAL: REPORTE DE UN CASO
}

\author{
Jesús Rojas-Jaimes ${ }^{1, a}$, Raul Castillo Cordova ${ }^{2, b}$, Delza Tárraga Gonzales ${ }^{1, a}$
}

\begin{abstract}
RESUMEN
La paracoccidiomicosis es una enfermedad micótica que puede comprometer el sistema nervioso central (SNC). Se presenta el caso de un paciente varón de 45 años, inmunocompetente, de la ciudad de Satipo, que desarrollo paracoccidiomicosis en el SNC sin punto de entrada e infección aparente. El paciente fue diagnosticado por imágenes con un granuloma cerebelar, posteriormente, se realiza una craneotomía de donde se obtiene tejido y secreción del absceso del granuloma, se procedió al diagnóstico histológico y microbiológico, respectivamente, donde se observó la presencia de levaduras en el corte histológico y el crecimiento del hongo en el cultivo. El paciente, inicialmente, recibe antifúngicos, luego adquiere una infección intrahospitalaria, recibe colistina y posteriormente fallece. El caso reportado pone énfasis en la importancia del diagnóstico temprano y correcto para la buena evolución de los casos de paracoccidiomicosis.
\end{abstract}

Palabras clave: Paracoccidioidomicosis; Sistema Nervioso Central; Paracoccidioides (fuente: DeCS BIREME)

\section{PARACOCCIDIOMICOSIS IN THE CENTRAL NERVOUS SYSTEM: A CASE REPORT}

\begin{abstract}
Paracoccidioidomycosis is a fungal disease which can compromise the central nervous system (CNS).We present a case of an immunocompetent 45 year old man from Satipo, Peru who developed paracoccidioidomycosis in the CNS without any apparent point of entry or infection. The patient was diagnosed by imaging with a cerebellar granuloma, followed by a craniotomy where tissue and secretion of the granuloma abscess was obtained. Histological and microbiological diagnoses were performed on the tissue and secretion, respectively. Yeasts were observed in the histological section and growth of the fungus in the culture. The patient initially received antifungals, then acquired an intrahospital infection, received colistin and subsequently died. The case report emphasizes the importance of early and correct diagnosis for good outcomes of paracoccidioidomycosis cases.
\end{abstract}

Key words: Paracoccidioidomycosis; Central Nervous System; Paracoccidioides (source: MeSH NLM).

\section{INTRODUCCIÓN}

La paracoccidiomicosis es un enfermedad micótica aguda o crónica con compromiso sistémico reportada en Centroamérica y Sudamérica, el agente involucrado en la enfermedad es conocido como Paracoccidioides brasiliensis, un hongo termodimórfico del género Paracoccidioides, del Phylum Ascomiceta, Clase Euromiceta, Orden Onygenales, Familia Onygenaceae. El agente es encontrado en zonas tropicales o semitropicales y la enfermedad se contrae por inhalación de conidios y micelios, cuya forma de diseminación es vía hematófaga y linfática, frecuentemente con compromiso gastrointestinal y oral afectando la mucosa y la lengua ${ }^{(1-5)}$.

La distribución geográfica se registra solo en América Latina, sobre todo en Brasil. En el Perú, la mayoría de los casos proceden de las regiones de Huánuco y Junín. Esta enfermedad es también conocida como "blastomicosis sudamericana" y se caracteriza por ser una micosis sistémica, granulomatosa crónica - subaguda, producida por el hongo dimórfico Paraccocidioides brasiliensis, el cual se adquiere por

\footnotetext{
Laboratorio de Microbiología del Hospital Guillermo Almenara Irigoyen. Lima, Perú.

Departamento de Infectología del Hospital Guillermo Almenara Irigoyen. Lima, Perú.

Biólogo, ${ }^{\mathrm{b}}$ médico Infectólogo.

Recibido: 29-06-14 Aprobado: 10-12-15
}

Citar como: Rojas-Jaimes J, Castillo Cordova R, Tárraga Gonzales D. Paracoccidiomicosis en el sistema nervioso central: reporte de un caso. Rev Peru Med Exp Salud Publica. 2015;32(1):183-6 


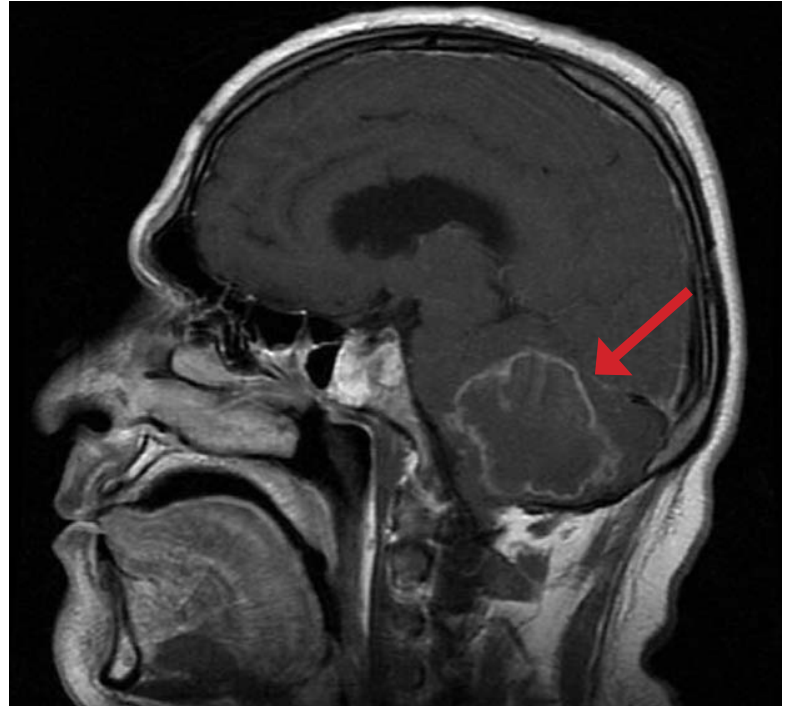

Figura 1. Resonancia magnética de vista lateral en donde se observa el cuerpo tumoral en zona cerebelar

inhalación de sus esporas en su fase micelial. En la mayoría de los casos la enfermedad aparece como una reactivación endógena que se desarrolla años después del contacto inicial con el hongo ${ }^{(6,7)}$. El hongo puede ser encontrado en animales silvestres como Dasypus novemcinctus, D. septemcinctus (armadillos), Procyon cancrivorus, Cavia aperea, Sphiggurus spinosus, Gallictis vittata, Eira barbara, y también en caninos ${ }^{(3)}$.

La respuesta inmune comienza con la interacción del patógeno y el tejido con una respuesta inmune adaptativa mediada por Th1. El contacto primario conduce a una acumulación de neutrófilos y formación de microabscesos ${ }^{(5)}$.

En el Perú se reportaron 111 casos de paracoccidiomicosis entre 1937 y 1965, siendo Junín la región con mayor prevalencia. Se presentó en mayor proporción en agricultores entre las edades de 41 a 50 años, siendo la mucocutánea en la zona oral la clínica de mayor prevalencia ${ }^{(8)}$. Respecto al diagnóstico, se efectúa mediante observaciones directas de cultivos, biopsias y pruebas con anticuerpos ${ }^{(9-11)}$. Es importante mencionar que el método del $\mathrm{KOH}$ es un método fácil y directo, especialmente en aspirados ganglionares para detección de la enfermedad, sin embargo, el cultivo y las biopsias de tejidos son los métodos de diagnóstico más destacados ${ }^{(12,13)}$

\section{REPORTE DEL CASO}

Hombre de 45 años de edad, agricultor, natural y procedente de Satipo; comenzó a presentar disartria y hemiparesia derecha en marzo de 2013; cefalea occipital sin horario en abril de 2013. El 5 de noviembre de 2013 presenta vómitos explosivos, mareos y cefalea intensa, por lo que se decidió realizarle una tomografía axial computarizada, donde se presenció hidrocefalia obstructiva (Figura 1). Se interna el 7 de noviembre de 2013, con un tiempo de enfermedad de 8 meses, el 9 de noviembre de 2013 es sometido a ventriculostomía endoscópica del tercer ventrículo. El examen clínico mostró borramiento del surco nasogeniano izquierdo, desviación de la comisura bucal a la derecha, dismetría derecha, síndrome cerebeloso y compromiso neurológico topográfico del hemisferio cerebelar derecho. La nosología fue: proceso expansivo cerebelar de etiología infecciosa vs neoplásico. El 29 de noviembre de 2013 se realizó una derivación ventrículo peritoneal (DVP); el 6 diciembre el paciente fue derivado a $\mathrm{UCl}$ neuroquirúrgica. $\mathrm{El} 8$ de diciembre de 2013 se realizó la craneotomía, el resultado fue: resección parcial de proceso expansivo indurado no vascularizado, fluido claro y espeso, granuloma por tuberculosis o micológico. El 9 de diciembre se obtuvo la secreción de absceso y se analizó por BK directo además de un cultivo, los cuales fueron negativos. Ese
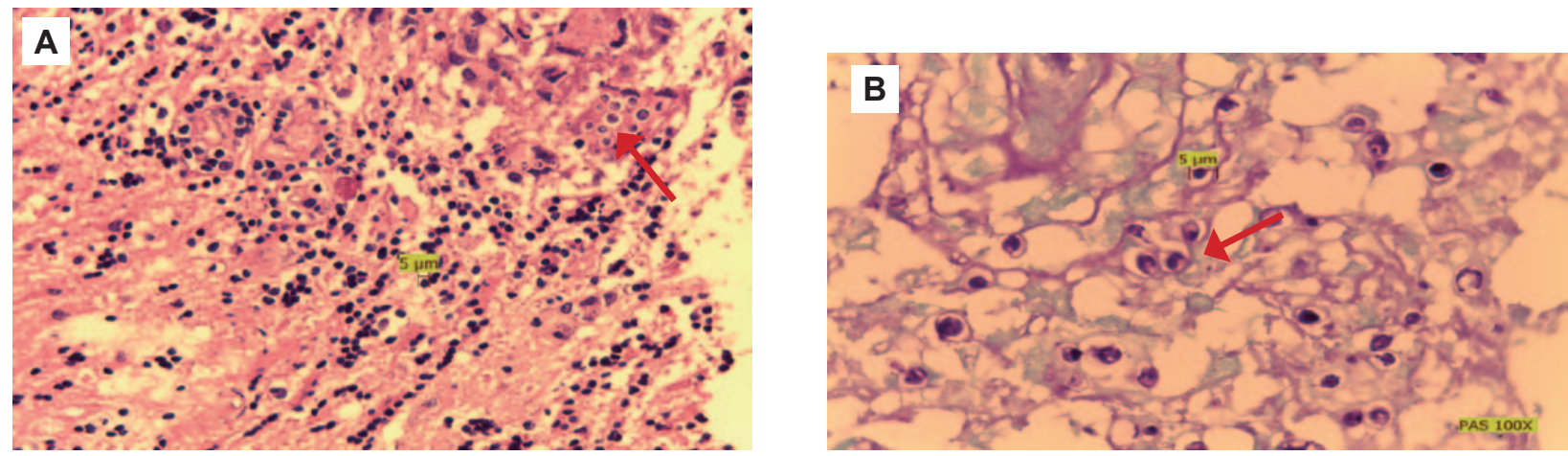

Figura 2. A) Tejido del granuloma teñido con eosina-hematoxilina. Se puede observar, levaduras en forma de "cabeza de Micky Mouse" (flecha), células infiltradas y tejido necrótico (aumento 400X). B) Histopatología con tinción PAS. Se observa tejido necrótico y levaduras (aumento 1000X) 
mismo día se obtuvo una biopsia que fue observada en histopatología con tinción hematoxilina-eosina, PAS y con nitrato de plata, se encontró levaduras por lo que se hizo el diagnóstico presuntivo de criptococosis (Figura 2). El 11 de diciembre se obtuvo secreción de absceso y se sembró en agar Sabouraoucloranfenicol el cual dio positivo para Paracoccidioides brasiliensis. Se inició el tratamiento con anfotericina B y posteriormente itraconazol; posteriormente el paciente adquirió una infección intrahospitalaria por Stenotrophomonas maltophilia MDR y Pseudomona aeruginosa panrresistente que le provocó neumonía, recibio colistina, pero posteriormente fallece.

\section{DISCUSIÓN}

Lasprincipalesmanifestaciones delaparacoccidiomicosis son el aumento de tamaño de ganglios y lesiones granulomatosas (1); esta enfermedad es adquirida por inhalación de los conidios producidos en la fase micelar que se transforman en levaduras, la mayoría de casos se da en los pulmones, su diseminación a otros tejidos es por vía hematógena; la incidencia que envuelve al sistema nervioso central es variable del 1,2 al $12,5 \%$. Las lesiones en el SNC están localizadas con frecuencia en el ámbito intraparenquimal, con uno o múltiples granulomas o abscesos que pueden involucrar a la meninge conduciendo a una aracnoiditis y simulando lesiones tumorales ${ }^{(4)}$. El compromiso en el sistema nervioso central provoca hipertensión intracraneal con formas seudotumorales (granulomas) en lóbulos parietales y frontales con daño parenquimal asociado con compromiso pulmonar y altas tasas de mortalidad, la neuroparacoccidiomicosis es casi exclusiva de la enfermedad crónica en adultos y puede desencadenar una meningoencefalitis

En el caso del paciente estudiado el diagnóstico inicial tomográfico fue la presencia de una tumoración con un tiempo de evolución sintomática de 8 meses, posteriormente, a través de una resonancia magnética se pudo determinar la presencia de un granuloma en la región cerebelar derecha. El granuloma produjo un efecto obstructivo con resultado de hipertensión intracraneal e hidrocefalia, por lo que el paciente necesitó ser intervenido quirúrgicamente para liberar la presión intracraneal. Fue necesario distinguir la paracoccidiomicosis de la tuberculosis, debido a que existe una clínica similar y es un factor de riesgo ${ }^{(1,3,14)}$.
Un reporte anterior describe a un paciente de 39 años que ingresa al servicio médico por dolores de cabeza y tremor de brazos durante tres meses; los exámenes físicos fueron normales. Los exámenes serológicos de doble difusión fueron negativos para Paracoccidioides brasiliensis; el examen de precipitación fue normal; la inmunofluorescencia indirecta fue normal; el examen de rayos $X$ mostró una infiltración micronodular parahiliar bilateral; la tomografía computarizada indicó un lesión cerebelar en el hemisferio derecho, se tomó muestra de líquido cefalorraquídeo para coloración Gram, Ziel Nielsen y cultivos, que resultaron negativos. Finalmente, la biopsia del corte histológico con tinción hematoxilina-eosina dio resultado positivo para granuloma por Paracoccidioides brasiliensis. Se realizó una intervención quirúrgica para extraer la tumoración, posteriormente se administró al paciente sulfametaxol $800 \mathrm{mg}$ más trimetropin $600 \mathrm{mg}$ dos veces al día, evolucionando satisfactoriamente ${ }^{(4)}$. Por el contrario, en nuestro estudio no se pudo localizar el punto de entrada del hongo o algún otro tejido involucrado. La paracoccidiomicosis se desarrolló básicamente en el SNC, por la derivación del hongo básicamente hacia el cerebro. La tomografía fue fundamental para localizar las lesiones, los exámenes histológicos de la biopsia permitieron encontrar al agente etiológico posterior a la intervención quirúrgica en la que es aconsejable extraer el tejido dañado y realizar un cultivo microbiológico de la biopsia o del líquido de absceso extraído.

Finalmente, cabe mencionar que según estudios previos los factores de riesgo a tomar en consideración para adquirir paracoccidiomicosis incluyen la actividad agrícola, residir en un lugar endémico y haber sufrido tuberculosis ${ }^{(1,3,14)}$. La tomografía axial computarizada del tórax mostró la neumonía Intrahospitalaria desarrollada, por cual el paciente falleció ${ }^{(15)}$.

Agradecimiento: al personal de Histopatología Clínica del Hospital Guillermo Almenara Irigoyen, que apoyó en el presente reporte de caso.

Contribuciones de autoría: JRJ, RCC, DTG participaron en la concepción y diseño del artículo, obtención de resultados. JRJ participó en el análisis e interpretación de datos, JRJ, RCC, DTG participaron en la redacción del artículo, revisión crítica y aprobación de su versión final.

Conflictos de interés: los autores declaran no tener conflictos de Interés.

Fuentes de financiamiento: autofinanciado. 


\section{REFERENCIAS BIBLIOGRÁFICAS}

1. Casquero J, Demarini J, Castillo M, Candella J, Zurita S. Nuevos casos de paracoccidiomicosis. Rev Peru Med Exp Salud Publica. $1998 ; 15(2): 12-7$.

2. Muñoz A, Chaparro E, Ferrufino J, Vasquez L. Apendicitis por Paracoccidioides brasiliensis. Rev Med Hered. 2006;17(1):58-60.

3. Marques SA. Paracoccidioidomycosis: epidemiological, clinical, diagnostic and treatment up-dating. An Bras Dermatol. 2013 SepOct;88(5):700-11. doi: 10.1590/ abd1806-4841.20132463.

4. Teive HA, Arruda WO, Ramina R, Meneses MS, Bleggi-Torres LF, TellesFilho FQ. Paracoccidioidomycosis granuloma simulating posterior fossa tumour., J R Soc Med. 1991;84(9):562-3.

5. Araújo V, Dias A, Borges A, Passador-Santos F, Henrique M, Ferrerira E, et al. Neutrophils in oral paracoccidioidomycosis and the involvement of Nrf2. PLoS One. 2013 Oct 24;8(10):e76976. doi: 10.1371/ journal.pone.0076976.
6. Brummer E, Castañeda E, Restrepo A. Para coccidioidomycosis: an update. Clin Microbiol Rev. 1993 Apr;6(2):89-117.

7. Cortez AC, de Souza JV, Sadahiro A, de Oliveira JA. Frequency and aetiology of dermatophytosis in children age 12 and under in the state of Amazonas, Brazil. Rev Iberoam Micol. 2012 OctDec;29(4):223-6. doi: 10.1016/j. riam.2012.02.004.

8. Burstein Z. Aspectos clínicos de la Blastomicosis sudamericana (Paracoccidioidomicosis) en el Perú. Rev Peru Med Exp Salud Publica. 2002;19(1):43-7.

9. Bejar V. Manual práctico de micología. Lima: Universidad Nacional Mayor de San Marcos; 2010.

10. San-Blas G. La micología molecular en la práctica médica del siglo XXI. Dermatología Venezolana. 2004;2004(1):4-8.

11. Walker S. Microbiología. México: McGraw-Hill Interamericana Editores; 1998.

12. Giglio P, Melgarejo C, Ramos C, Feria K, Puell L, Salomón M. et al. Paracoccidioidomicosis juvenil diseminada. Folia Dermatol Peru. 2008;19(3):121-6.

13. Subauste A, Castillo M, Torres R. Paracoccidioidomicosis pediátrica. Folia Dermatol Peru. 1997;8(4):30-8.

14. Bellissimo-Rodrigues F, Machado AA, Martinez R. Paracoccidioidomycosis epidemiological features of a 1,000-cases series from a hyperendemic area on the southeast of Brazil. Am J Trop Med Hyg. 2011 Sep;85(3):54650. doi: 10.4269/ajtmh.2011.11-0084.

15. Salina MA, Shikanai-Yasuda MA, Mendes RP, Barraviera B, Mendes Giannini MJ. Detection of circulating Paracoccidioides brasiliensis antigen in urine of Paracoccidioidomycosis patients before and during treatment. J Clin Microbiol. 1998 Jun;36(6):1723-8.

Correspondencia: Jesús Rojas Jaimes

Dirección: Av. Cerro de Pasco 319, Comas,

Perú.

Teléfono: 993638840

Correo electrónico: jesus.rojas.jaimess@ gmail.com

\section{Consulte las ediciones anteriores de la Revista Peruana de Medicina Experimental y Salud Pública en www.scielosp.org}

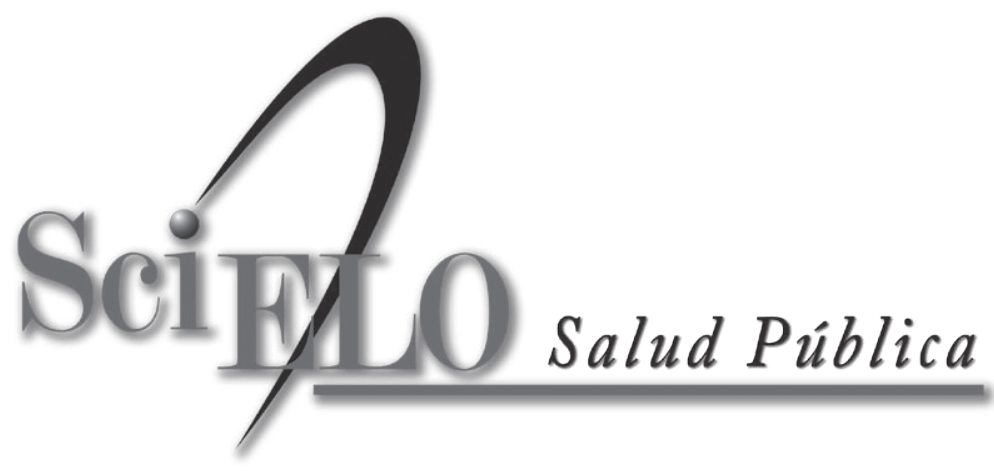

